\title{
Prognostic value of postoperative C-reactive protein elevation versus complication occurrence: a multicenter validation study
}

\author{
Yukinori Kurokawa ${ }^{1} \cdot$ Kotaro Yamashita $^{1} \cdot$ Ryohei Kawabata $^{2} \cdot$ Junya Fujita $^{3} \cdot$ Hiroshi Imamura $^{4} \cdot$ Atsushi Takeno $^{5}$. \\ Tsuyoshi Takahashi ${ }^{1} \cdot$ Makoto Yamasaki $^{1} \cdot$ Hidetoshi Eguchi $^{1} \cdot$ Yuichiro Doki $^{1}$
}

Received: 23 January 2020 / Accepted: 6 April 2020 / Published online: 20 April 2020

(c) The International Gastric Cancer Association and The Japanese Gastric Cancer Association 2020

\begin{abstract}
Background Several studies have shown that postoperative complications worsen the prognosis of patients with malignancies. However, our previous study showed that C-reactive protein (CRP) elevation over $12 \mathrm{mg} / \mathrm{dL}$ was a more reliable prognostic indicator than complication occurrence. This large-scale, multicenter validation study aimed to confirm the prognostic value of postoperative CRP elevation in resectable gastric cancer.

Methods Data of 1456 patients with pT2-T4 gastric cancer who underwent R0 resection were collected from 21 institutions. The prognostic value of the highest postoperative serum level of CRP $\left(\mathrm{CRP}_{\max }\right)$ during hospitalization was evaluated using the Kaplan-Meier method. The prognostic independence of $\mathrm{CRP}_{\max }$ with assessed with a Cox multivariate analysis of recurrence-free survival (RFS).

Results RFS in the high $\mathrm{CRP}_{\max }(\geq 12 \mathrm{mg} / \mathrm{dL})$ group was significantly worse than that in the low $\mathrm{CRP}_{\max }(<12 \mathrm{mg} / \mathrm{dL})$ group (log-rank $P=0.002)$. The recurrence pattern showed that liver metastasis occurred more frequently in the high $\mathrm{CRP}_{\max }$ group (9.2\%) than in the low $\mathrm{CRP}_{\max }$ group (4.7\%) $(P=0.001)$. In patients without intra-abdominal infectious complications, the high $\mathrm{CRP}_{\max }$ group showed significantly worse RFS than the low $\mathrm{CRP}_{\max }$ group (log-rank $\left.P=0.026\right)$. In patients with intraabdominal infectious complications, the high $\mathrm{CRP}_{\text {max }}$ group had worse RFS than the low $\mathrm{CRP}_{\text {max }}$ group, but this difference was not significant (log-rank $P=0.075)$. Cox multivariate analysis with 13 covariables showed that $\mathrm{CRP}_{\max }(P=0.043)$ was an independent prognostic factor, but postoperative complications were not $(P=0.387)$.

Conclusion Postoperative CRP elevation was a better predictor of prognosis in patients with gastric cancer than the occurrence of intra-abdominal infectious complications.
\end{abstract}

Keywords Gastric cancer · Gastrectomy $\cdot$ C-reactive protein $\cdot$ Postoperative complication

Yukinori Kurokawa

ykurokawa@gesurg.med.osaka-u.ac.jp

1 Department of Gastroenterological Surgery, Osaka University Graduate School of Medicine, 2-2, Yamadaoka, Suita, Osaka 565-0871, Japan

2 Department of Surgery, Osaka Rosai Hospital, Osaka, Japan

3 Department of Surgery, Sakai City Medical Center, Osaka, Japan

4 Department of Surgery, Toyonaka Municipal Hospital, Osaka, Japan

5 Department of Surgery, Kansai Rosai Hospital, Hyogo, Japan

\section{Introduction}

Gastric cancer is a common malignancy worldwide [1]. While surgical resection is the most effective curative treatment, many patients still experience recurrence [2,3]. Pathological TNM staging is the most useful indicator of prognosis after surgery [4], but it is still insufficient. We, therefore, need additional prognostic indicators to complement the TNM stage and to determine optimal postoperative strategies, including adjuvant treatment, in patients with resectable gastric cancer.

Several studies have shown that postoperative complications worsen the prognosis of patients with malignancies [5-8]. Such complications increase the levels of inflammatory cytokines such as interleukin-6 (IL-6) and may lead to the proliferation of residual cancer cells [9-11]. These 
outcomes can be caused by excessive surgical stress even if postoperative complications do not occur. Indeed, several randomized controlled trials demonstrated that compared with standard surgery, extended surgery worsened the prognosis of patients with gastroesophageal cancer [12-14]. $\mathrm{C}$-reactive protein (CRP) is the most common indicator of systemic inflammation and is closely correlated with serum IL-6 levels $[15,16]$. We previously showed that CRP elevation over $12 \mathrm{mg} / \mathrm{dL}$ was a more reliable indicator of worse recurrence-free survival (RFS) in gastric cancer patients than the occurrence of postoperative complications [17]. However, since the study was conducted at a single institution, we considered it important to validate the usefulness of CRP elevation in a large-scale multicenter study. Thus, we conducted this multicenter validation study to confirm the prognostic value of postoperative CRP elevation with over 1400 patients who underwent surgical resection of pT2-T4 gastric cancer.

\section{Methods}

\section{Patients}

The eligible population in this study consisted of patients with pathological $\mathrm{T} 2$ or greater advanced gastric cancer who underwent R0 resection between January 2008 and December 2010 at 21 institutions belonging to the Clinical Study Group of Osaka University. Exclusion criteria were as follows: synchronous coexisting cancer, metachronous cancer within 5 years, and preoperative treatment. TNM staging was performed according to the 7th edition of the Union for International Cancer Control (UICC) TNM classification [4]. In principle, gastrectomy, lymph node dissection, adjuvant chemotherapy, and postoperative follow-up were carried out according to the 3rd edition of the Japanese Gastric Cancer Treatment Guidelines [18]. This study was approved by the institutional review board of Osaka University Hospital (No. 15001).

\section{Statistics}

The primary endpoint of this study was the association between prognosis and the highest postoperative serum level of CRP $\left(\mathrm{CRP}_{\max }\right)$ during the hospital stay. Statistical differences were also examined after adjustment for the presence or absence of postoperative complications. Postoperative complications were assessed according to the Clavien-Dindo classification $[19,20]$. We defined postoperative complications as intra-abdominal infectious complications of Grade II or higher, in accordance with a similar study in the field of gastric cancer surgery [8].
Associations between clinicopathological factors and $\mathrm{CRP}_{\max }$ were compared using the chi-square test for categorical variables and the Mann-Whitney $U$ test for continuous variables. Recurrence-free survival (RFS) was defined as the time from surgery to either the first recurrence or death from any cause. Overall survival (OS) was defined as the time from surgery to death from any cause. RFS and OS curves were estimated using the Kaplan-Meier method, and survival differences were compared using the log-rank test. Cox proportional hazards models were used for both univariate and multivariate analyses. $P$ values less than 0.05 were considered statistically significant. Statistical analyses were performed with the SPSS statistical package, version 24.0 (SPSS, Chicago, IL, USA).

\section{Results}

\section{Study population}

A total of 1456 patients with pT2-T4 gastric cancer were enrolled in this study (Table 1). The median $\mathrm{CRP}_{\max }$ levels were $11.75 \mathrm{mg} / \mathrm{dL}$ (range, $0.17-43.10 \mathrm{mg} / \mathrm{dL}$ ) in the whole patients, $7.95 \mathrm{mg} / \mathrm{dL}$ (range, $0.17-11.96 \mathrm{mg} / \mathrm{dL}$ ) in the low $\mathrm{CRP}_{\max }$ group, and $17.49 \mathrm{mg} / \mathrm{dL}$ (range, $12.00-43.10 \mathrm{mg}$ / $\mathrm{dL}$ ) in the high $\mathrm{CRP}_{\max }$ group. Male patients and patients with a high body mass index were more common in the high $\mathrm{CRP}_{\text {max }}(\geq 12 \mathrm{mg} / \mathrm{dL})$ group than in the low $\mathrm{CRP}_{\text {max }}$ $(<12 \mathrm{mg} / \mathrm{dL})$ group. The high $\mathrm{CRP}_{\max }$ group included more patients with differentiated histological type, total gastrectomy, long operation time, large amount of intraoperative blood loss, and advanced pT tumors than the low $\mathrm{CRP}_{\max }$ group. Postoperative complications occurred in 18 patients (2.4\%) in the low $\mathrm{CRP}_{\text {max }}$ group and 159 patients (22.5\%) in the high $\mathrm{CRP}_{\max }$ group. The most frequent complications were anastomotic leakage in 74 patients $(5.1 \%)$, abdominal abscess in 65 (4.5\%), and pancreatic fistula in $59(4.1 \%)$.

\section{Kaplan-Meier survival analysis}

At the median follow-up duration of 61.0 months for the censored cases, the RFS in the high $\mathrm{CRP}_{\text {max }}$ group was significantly worse than that in the low $\mathrm{CRP}_{\text {max }}$ group (log-rank $P=0.002$ ) (Fig. 1a). The hazard ratio (HR) of recurrence in the high $\mathrm{CRP}_{\max }$ group was 1.32 (95\% CI, 1.11-1.56), and the 5-year RFS rates were $59.8 \%$ in the high CRP $_{\text {max }}$ group and $67.1 \%$ in the low $\mathrm{CRP}_{\max }$ group. The OS also showed a significant difference between the two groups (log-rank $P<0.001$ ). The HR of death in the high $\mathrm{CRP}_{\max }$ group was 1.43 (95\% CI, 1.18-1.72), and the 5-year OS rates were $65.5 \%$ in the high $\mathrm{CRP}_{\max }$ group and $74.6 \%$ in the low $\mathrm{CRP}_{\max }$ group. In terms of recurrence pattern, only liver metastasis occurred significantly more frequently in the high 
Table 1 Patient characteristics according to the peak serum CRP concentration $\left(\mathrm{CRP}_{\max }\right)$

\begin{tabular}{|c|c|c|c|}
\hline & $\begin{array}{l}\text { Low } \text { CRP }_{\max } \\
(<12 \mathrm{mg} / \mathrm{dL}) \\
(n=749)\end{array}$ & $\begin{array}{l}\text { High } \mathrm{CRP}_{\max } \\
(\geq 12 \mathrm{mg} / \mathrm{dL}) \\
(n=707)\end{array}$ & $P$ value \\
\hline Age (median, range), (years) & $68(24-94)$ & $70(26-92)$ & 0.008 \\
\hline Sex & & & $<0.001$ \\
\hline Male & $439(58.6 \%)$ & $568(80.3 \%)$ & \\
\hline Female & $310(41.4 \%)$ & $139(19.7 \%)$ & \\
\hline Body mass index (median, range), $\left(\mathrm{kg} / \mathrm{m}^{2}\right)$ & $21.5(12.9-31.6)$ & $22.9(12.0-33.6)$ & $<0.001$ \\
\hline Histological type & & & 0.024 \\
\hline Differentiated & $334(44.6 \%)$ & $357(50.5 \%)$ & \\
\hline Undifferentiated & $415(55.4 \%)$ & $350(49.5 \%)$ & \\
\hline Approach & & & 0.100 \\
\hline Open & $645(86.1 \%)$ & $629(89.0 \%)$ & \\
\hline Laparoscopic & $104(13.9 \%)$ & $78(11.0 \%)$ & \\
\hline Type of gastrectomy & & & $<0.001$ \\
\hline Subtotal & $518(69.2 \%)$ & $413(58.4 \%)$ & \\
\hline Total & $231(30.8 \%)$ & $294(41.6 \%)$ & \\
\hline Lymphadenectomy & & & 0.125 \\
\hline$<\mathrm{D} 2$ & $163(21.8 \%)$ & $131(18.5 \%)$ & \\
\hline$\geq \mathrm{D} 2$ & $586(78.2 \%)$ & $576(81.5 \%)$ & \\
\hline Operation time (median, range), (min) & $223(65-499)$ & $249(100-535)$ & $<0.001$ \\
\hline Intraoperative blood loss (median, range), $(\mathrm{mL})$ & $250(0-3500)$ & $400(0-6340)$ & $<0.001$ \\
\hline pT status & & & 0.013 \\
\hline $\mathrm{T} 2$ & $240(32.0 \%)$ & $177(25.0 \%)$ & \\
\hline $\mathrm{T} 3$ & $251(33.5 \%)$ & $263(37.2 \%)$ & \\
\hline $\mathrm{T} 4$ & $258(34.4 \%)$ & $267(37.8 \%)$ & \\
\hline pN status & & & 0.304 \\
\hline No & $311(41.5 \%)$ & $264(37.3 \%)$ & \\
\hline N1 & $154(20.6 \%)$ & $142(20.1 \%)$ & \\
\hline $\mathrm{N} 2$ & $139(18.6 \%)$ & $149(21.1 \%)$ & \\
\hline N3 & $145(19.4 \%)$ & $152(21.5 \%)$ & \\
\hline Adjuvant chemotherapy & & & 0.859 \\
\hline Absent & $334(44.6 \%)$ & $312(44.1 \%)$ & \\
\hline Present & $415(55.4 \%)$ & $395(55.9 \%)$ & \\
\hline Postoperative complications & & & $<0.001$ \\
\hline Absent & $731(97.6 \%)$ & $548(77.5 \%)$ & \\
\hline Present & $18(2.4 \%)$ & $159(22.5 \%)$ & \\
\hline
\end{tabular}

pT/N status and pStage were according to the Union for International Cancer Control (UICC) TNM classification of malignant tumours (7th edition). Postoperative complications mean only intra-abdominal infectious complications of Grade II or higher (Clavien-Dindo classification)
$\mathrm{CRP}_{\text {max }}$ group (9.2\%) than in the low $\mathrm{CRP}_{\max }$ group $(4.7 \%)$ $(P=0.001)$ (Table 2). Even in the patients who did not have a recurrence in the liver, the high $\mathrm{CRP}_{\max }$ group showed significantly worse RFS and OS than the low $\mathrm{CRP}_{\max }$ group (log-rank $P=0.044, P=0.008$, respectively).

Similarly, the RFS was significantly worse in patients who had postoperative complications than in those who did not (log-rank $P=0.016$ ) (Fig. 1b). We conducted a subgroup analysis to evaluate the prognostic value of postoperative CRP elevation independently of the presence or absence of postoperative complications. Among patients with no postoperative complications, the RFS was significantly worse in the high $\mathrm{CRP}_{\max }$ group than in the low $\mathrm{CRP}_{\max }$ group (log-rank $P=0.026$; Fig. $2 \mathrm{a}$ ). Among patients with postoperative complications, the high $\mathrm{CRP}_{\max }$ group had worse RFS than the low $\mathrm{CRP}_{\max }$ group, but this difference was not significant (log-rank $P=0.075$; Fig. 2b).

\section{Cox multivariate analysis}

To assess the prognostic value of $\mathrm{CRP}_{\max }$ independently of other confounding factors, we conducted a Cox multivariate 


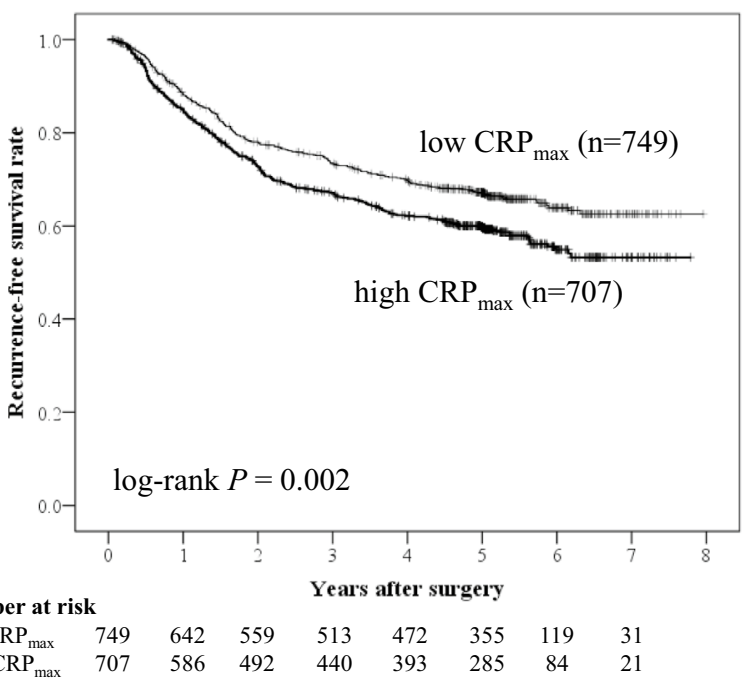

b

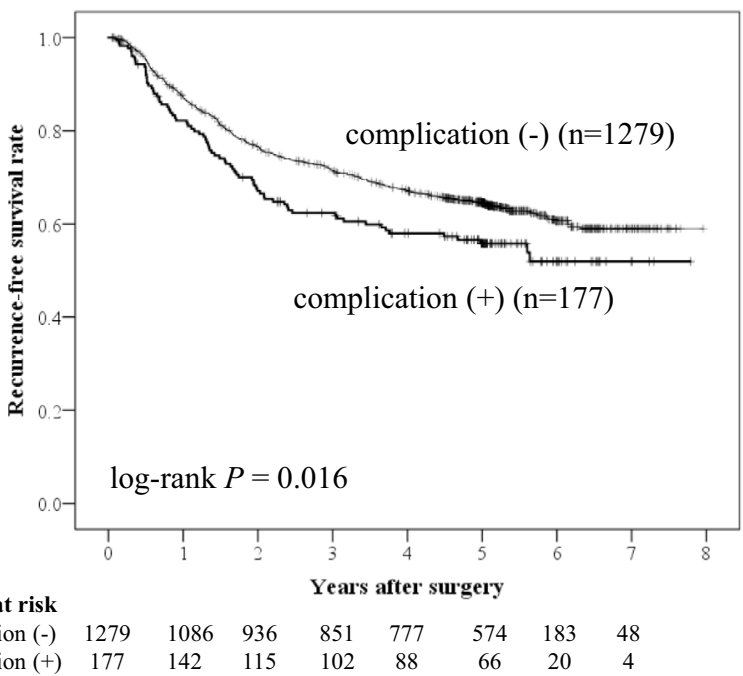

Fig. 1 Kaplan-Meier recurrence-free survival between the low $(<12 \mathrm{mg} / \mathrm{dL})$ and high $\mathrm{CRP}_{\max }(\geq 12 \mathrm{mg} / \mathrm{dL})$ groups (a) and between patients without and with postoperative complications (b)

Table 2 Patterns of recurrence

\begin{tabular}{lccc}
\hline Variable & $\begin{array}{l}\text { Low CRP } \\
(n=749)\end{array}$ & $\begin{array}{l}\text { High CRP } \\
(n=707)\end{array}$ & $P$ value \\
\hline Recurrence & $196(26.2 \%)$ & $231(32.7 \%)$ & 0.006 \\
Peritoneum & $88(11.7 \%)$ & $79(11.2 \%)$ & 0.731 \\
Liver & $35(4.7 \%)$ & $65(9.2 \%)$ & 0.001 \\
Lymph node & $68(9.1 \%)$ & $59(8.3 \%)$ & 0.620 \\
Local & $5(0.7 \%)$ & $11(1.6 \%)$ & 0.104 \\
\hline
\end{tabular}

$\mathbf{a}$

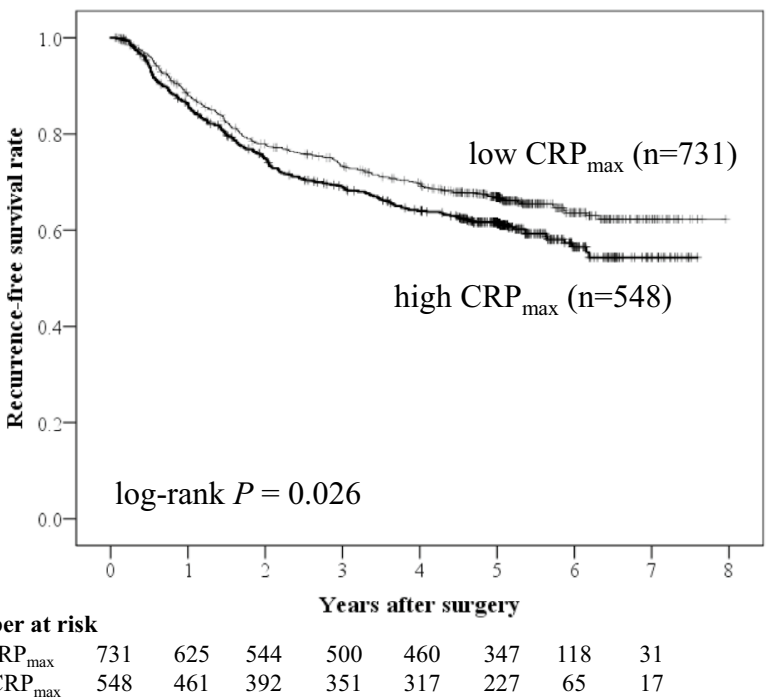

analysis of RFS with 13 covariables (Table 3 ). The multivariate analysis identified $\mathrm{CRP}_{\max }(P=0.043)$ as an independent prognostic factor, along with age $(P<0.001)$, body mass index $(P=0.008)$, type of gastrectomy $(P=0.006)$, pT status $(P<0.001)$, pN status $(P<0.001)$, and adjuvant chemotherapy $(P=0.004)$. By contrast, the postoperative complication was not a significant factor $(P=0.387)$.

b

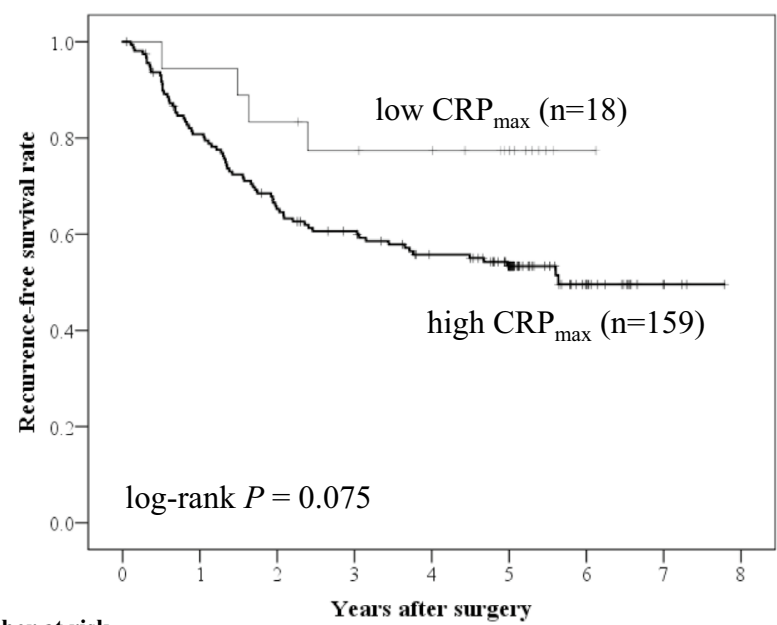

Number at risk

$\begin{array}{llllllccc}\text { low } \mathrm{CRP}_{\max } & 18 & 17 & 15 & 13 & 12 & 8 & 1 & 0\end{array}$

Fig. 2 Kaplan-Meier recurrence-free survival between the low $(<12 \mathrm{mg} / \mathrm{dL})$ and high $\mathrm{CRP}_{\max }(\geq 12 \mathrm{mg} / \mathrm{dL})$ groups in patients without $(\mathbf{a})$ and with (b) postoperative complications 
Table 3 Cox multivariate analysis of prognostic factors associated with recurrence-free survival after gastric surgery

\begin{tabular}{|c|c|c|}
\hline Variables & HR $(95 \%$ CI $)$ & $P$ value \\
\hline \multicolumn{3}{|l|}{ Age } \\
\hline$\geq 70$ years & $1.54(1.28-1.86)$ & $<0.001$ \\
\hline \multicolumn{3}{|l|}{ Sex } \\
\hline Male & $1.05(0.86-1.29)$ & 0.610 \\
\hline \multicolumn{3}{|l|}{ Body mass index } \\
\hline$<22 \mathrm{~kg} / \mathrm{m}^{2}$ & $1.27(1.06-1.52)$ & 0.008 \\
\hline \multicolumn{3}{|l|}{ Histological type } \\
\hline Differentiated & $1.12(0.94-1.34)$ & 0.218 \\
\hline \multicolumn{3}{|l|}{ Approach } \\
\hline Open & $1.21(0.87-1.67)$ & 0.255 \\
\hline \multicolumn{3}{|c|}{ Type of gastrectomy } \\
\hline Total & $1.29(1.07-1.54)$ & 0.006 \\
\hline \multicolumn{3}{|c|}{ Lymph node dissection } \\
\hline D2 & $1.19(0.95-1.48)$ & 0.127 \\
\hline \multicolumn{3}{|l|}{ Operation time } \\
\hline$\geq 240 \min$ & $1.05(0.86-1.27)$ & 0.631 \\
\hline \multicolumn{3}{|l|}{ Blood loss } \\
\hline$\geq 320 \mathrm{~mL}$ & $1.01(0.83-1.24)$ & 0.913 \\
\hline \multicolumn{3}{|l|}{ pT status } \\
\hline $\mathrm{T} 4$ & $2.05(1.70-2.46)$ & $<0.001$ \\
\hline \multicolumn{3}{|l|}{ pN status } \\
\hline $\mathrm{N} 1-\mathrm{N} 3$ & $2.79(2.20-3.53)$ & $<0.001$ \\
\hline \multicolumn{3}{|c|}{ Adjuvant chemotherapy } \\
\hline No & $1.37(1.11-1.70)$ & 0.004 \\
\hline \multicolumn{3}{|c|}{ Postoperative complication } \\
\hline Yes & $1.12(0.86-1.46)$ & 0.387 \\
\hline \multicolumn{3}{|l|}{$\mathrm{CRP}_{\max }$} \\
\hline$\geq 12 \mathrm{mg} / \mathrm{dL}$ & $1.21(1.01-1.47)$ & 0.043 \\
\hline
\end{tabular}

\section{Discussion}

This large-scale, multicenter study validated our initial finding that postoperative CRP elevation was an independent prognostic factor in patients who underwent surgical resection of pT2-T4 gastric cancer. The occurrence of postoperative complications was a significant prognostic factor in a univariate Kaplan-Meier analysis but not in a Cox multivariate analysis, indicating that $\mathrm{CRP}_{\max }$ is a comprehensive prognostic indicator that takes into account the influence of postoperative complications. Moreover, in terms of objectivity, $\mathrm{CRP}_{\max }$ has a higher prognostic value than complication occurrence.

Several previous studies in various fields of cancer surgery showed that the occurrence of postoperative complications worsened patient prognosis [5-8]. Salvans et al. reported that postoperative peritoneal fluid obtained from patients with an anastomotic leak or intra-abdominal abscess enhanced both cell migration and cell invasion capacities in in vitro assays [9]. They concluded that intra-abdominal infectious complications enhance the invasiveness of residual tumor cells after surgery, leading to recurrence. Increased proinflammatory mediators such as interleukin-6 (IL-6) may play an important role in promoting proliferation and migration of residual cancer cells [21, 22]. Kubota et al. reported that postoperative complications of Grade II or higher were a significant prognostic indicator in pStage II and III but not pStage I gastric cancer [23]. However, their study was retrospectively conducted at a single institution, and more than half of their patients had early (pT1) gastric cancer. By contrast, in this study we enrolled a much larger number of patients with advanced (pT2-T4) gastric cancer. Our multivariate analysis revealed that the occurrence of postoperative complications was not an independent prognostic indicator, and that instead it was a confounding factor for CRP elevation.

CRP elevation is a comprehensive indicator of postoperative inflammation status, which in turn reflects the levels of inflammatory cytokines such as IL-6 [15, 16]. Even if postoperative complications do not occur, excessive surgical stress can result in the production of inflammatory cytokines, which leads to the promotion of adhesion of circulating tumor cells to the vascular endothelium of distant organs by enhancing the E-selectin expression [24-27]. This mechanism may explain the reason why liver not peritoneal metastasis occurred significantly more frequently in the high $\mathrm{CRP}_{\max }$ group in our study. Extended surgery that causes excessive surgical stress may worsen both patient prognosis and quality of life. Thus, minimally invasive surgery that does not increase the occurrence of postoperative complications is ideal in patients with cancer.

Recently, several complex prognostic indicators have been reported, including CRP. The Glasgow prognostic score, which uses CRP and albumin, is a well-known prognostic marker in various kinds of cancer surgeries [28]. The $\mathrm{CRP} /$ albumin ratio (CAR) was also reported to be a useful prognostic marker in hepatocellular carcinoma [29]. However, most studies using these complex indicators or CRP alone examined only the prognostic value of the preoperative CRP level [28-31]. Preoperative CRP mainly reflects oncological status, whereas postoperative CRP reflects both oncological status and the inflammatory response after surgery, and may, therefore, be more useful for predicting patient prognosis [32]. A large-scale study comparing the prognostic values of postoperative CRP alone with complex indicators that include postoperative CRP is warranted in the future.

This study has several limitations. First, it was a retrospective study and was therefore prone to selection bias, although the number of patients was very large. To reduce bias as much as possible, we collected the data of consecutive patients who underwent R0 resection between January 
2008 and December 2010 at 21 institutions. Second, no consensus guidelines currently exist for determining the type of complications that affect prognosis. However, a metaanalysis showed the relationship between complications and reduced survival was stronger when only infectious complications were considered as compared with all complications [32]. In this study, we, therefore, assessed intra-abdominal infectious complications in accordance with a previous study in gastric cancer surgery [8]. Third, the days of routine CRP examination were not fixed in this study, and we used the highest postoperative serum level of CRP during the hospital stay as a representative value of postoperative CRP elevation. However, CRP level at the fixed date after surgery may be better considering the simplicity. Indeed, Watt et al. examined the prognostic values of CRP and albumin levels on days 3 or 4 after colorectal surgery [33]. We actually confirmed that most of the participating institutions routinely examined CRP levels either on days 2-4. We hope future studies will evaluate which is a better prognostic indicator $\mathrm{CRP}_{\max }$ or CRP level at the fixed date.

In conclusion, postoperative CRP elevation was a much more effective predictor of prognosis in patients with gastric cancer than the occurrence of intra-abdominal infectious complications. CRP ${ }_{\max }$ over $12 \mathrm{mg} / \mathrm{dL}$ may be a useful complement to the TNM stage in determining the indications for adjuvant treatment to improve the prognosis of gastric cancer patients more. Surgeons should ideally avoid increasing postoperative CRP levels by reducing excessive surgical stress and postoperative complications.

Funding The authors declare that this study was not funded.

\section{Compliance with ethical standards}

Conflict of interest The authors declare that they have no conflicts of interest.

Ethical approval All procedures performed in studies involving human participants were in accordance with the ethical standards of the institutional research committee and with the 1964 Helsinki Declaration and its later amendments.

Informed consent Consent to participate was not considered necessarily.

\section{References}

1. International Agency for Research on Cancer. GLOBOCAN 2018: cancer incidence and mortality worldwide. https://gco. iarc.fr/today/data/factsheets/cancers/39-All-cancers-fact-sheet .pdf. Accessed 18 Apr 2020.

2. Hashimoto T, Kurokawa Y, Mori M, Doki Y. Update on the treatment of gastric cancer. JMA J. 2018;1:40-9.
3. Kurokawa Y, Doki Y, Mizusawa J, et al. Bursectomy versus omentectomy alone for resectable gastric cancer (JCOG1001): a phase 3, open-label, randomised controlled trial. Lancet Gastroenterol Hepatol. 2018;3:460-8.

4. Sobin LH, Gospodarowicz MK, Wittekind CH. TNM classification of malignant tumours. 7th ed. New York: Wiley-Blackwell; 2009.

5. Artinyan A, Orcutt ST, Anaya DA, et al. Infectious postoperative complications decrease long-term survival in patients undergoing curative surgery for colorectal cancer: a study of 12,075 patients. Ann Surg. 2015;261:497-505.

6. Kataoka K, Takeuchi H, Mizusawa J, et al. Prognostic impact of postoperative morbidity after esophagectomy for esophageal cancer: exploratory analysis of JCOG9907. Ann Surg. 2017;265:1152-7.

7. Murthy BL, Thomson CS, Dodwell D, et al. Postoperative wound complications and systemic recurrence in breast cancer. Br J Cancer. 2007;97:1211-7.

8. Tokunaga $\mathrm{M}$, Tanizawa $\mathrm{Y}, \mathrm{Bando} \mathrm{E}$, et al. Poor survival rate in patients with postoperative intra-abdominal infectious complications following curative gastrectomy for gastric cancer. Ann Surg Oncol. 2013;20:1575-83.

9. Salvans S, Mayol X, Alonso S, et al. Postoperative peritoneal infection enhances migration and invasion capacities of tumor cells in vitro: An insight into the association between anastomotic leak and recurrence after surgery for colorectal cancer. Ann Surg. 2014;260:939-44.

10. Szczepanik AM, Scislo L, Scully T, et al. IL-6 serum levels predict postoperative morbidity in gastric cancer patients. Gastric Cancer. 2011;14:266-73.

11. Taniguchi Y, Kurokawa Y, Hagi T, et al. Methylprednisolone inhibits tumor growth and peritoneal seeding induced by surgical stress and postoperative complications. Ann Surg Oncol. 2019;26:2831-8.

12. Sasako M, Sano T, Yamamoto S, et al. Left thoracoabdominal approach versus abdominal-transhiatal approach for gastric cancer of the cardia or subcardia: a randomised controlled trial. Lancet Oncol. 2006;7:644-51.

13. Kurokawa Y, Sasako M, Sano T, et al. Ten-year follow-up results of a randomized clinical trial comparing left thoracoabdominal and abdominal transhiatal approaches to total gastrectomy for adenocarcinoma of the oesophagogastric junction or gastric cardia. Br J Surg. 2015;102:341-8.

14. Sano T, Sasako M, Mizusawa J, et al. Randomized controlled trial to evaluate splenectomy in total gastrectomy for proximal gastric carcinoma. Ann Surg. 2017;265:277-83.

15. Hirano T, Akira S, Taga T, Kishimoto T. Biological and clinical aspects of interleukin 6. Immunol Today. 1990;11:443-9.

16. Ikeda U, Ohkawa F, Seino Y, et al. Serum interleukin 6 levels become elevated in acute myocardial infarction. J Mol Cell Cardiol. 1992;24:579-84.

17. Saito T, Kurokawa Y, Miyazaki Y, et al. Which is a more reliable indicator of survival after gastric cancer surgery: postoperative complication occurrence or C-reactive protein elevation? J Surg Oncol. 2015;112:894-9.

18. Japanese Gastric Cancer Association. Japanese gastric cancer treatment guidelines 2010 (ver. 3). Gastric Cancer. 2011;14:113-23.

19. Dindo D, Demartines N, Clavien PA. Classification of surgical complications. Ann Surg. 2004;240:205-13.

20. Katayama H, Kurokawa Y, Nakamura K, et al. Extended Clavien-Dindo classification of surgical complications: Japan Clinical Oncology Group postoperative complications criteria. Surg Today. 2016;46:668-85. 
21. Lin MT, Lin BR, Chang CC, et al. IL-6 induces AGS gastric cancer cell invasion via activation of the c-Src/RhoA/ROCK signaling pathway. Int J Cancer. 2007;120:2600-8.

22. Foran E, Garrity-Park MM, Mureau C, et al. Upregulation of DNA methyltransferase-mediated gene silencing, anchorage-independent growth, and migration of colon cancer cells by interleukin- 6 . Mol Cancer Res. 2010;8:471-81.

23. Kubota T, Hiki N, Sano T, et al. Prognostic significance of complications after curative surgery for gastric cancer. Ann Surg Oncol. 2014;21:891-8.

24. Barthel SR, Gavino JD, Descheny L, et al. Targeting selectins and selectin ligands in inflammation and cancer. Expert Opin Ther Targets. 2007;11:1473-91.

25. Tremblay PL, Huot J, Auger FA. Mechanisms by which E-selectin regulates diapedesis of colon cancer cells under flow conditions. Cancer Res. 2008;68:5167-76.

26. Hiratsuka S, Goel S, Kamoun WS, et al. Endothelial focal adhesion kinase mediates cancer cell homing to discrete regions of the lungs via E-selectin up-regulation. Proc Natl Acad Sci USA. 2011;108:3725-30.

27. Nojiri T, Hosoda H, Tokudome T, et al. Atrial natriuretic peptide prevents cancer metastasis through vascular endothelial cells. Proc Natl Acad Sci USA. 2015;112:4086-91.

28. McMillan DC. Systemic inflammation, nutritional status and survival in patients with cancer. Curr Opin Clin Nutr Metab Care. 2009;12:223-6.
29. Kinoshita A, Onoda H, Imai N, et al. The C-reactive protein/albumin ratio, a novel inflammation-based prognostic score, predicts outcomes in patients with hepatocellular carcinoma. Ann Surg Oncol. 2015;22:803-10.

30. Kubota T, Hiki N, Nunobe S, et al. Significance of the inflammation-based Glasgow prognostic score for short- and long-term outcomes after curative resection of gastric cancer. J Gastrointest Surg. 2012;16:2037-44.

31. Jiang $X$, Hiki N, Nunobe $S$, et al. Prognostic importance of the inflammation-based Glasgow prognostic score in patients with gastric cancer. Br J Cancer. 2012;107:275-9.

32. Pucher PH, Aggarwal R, Qurashi M, et al. Meta-analysis of the effect of postoperative in-hospital morbidity on long-term patient survival. Br J Surg. 2014;101:1499-508.

33. Watt DG, McSorley ST, Park JH, Horgan PG, McMillan DC. A postoperative systemic inflammation score predicts short- and long-term outcomes in patients undergoing surgery for colorectal cancer. Ann Surg Oncol. 2017;24:1100-9.

Publisher's Note Springer Nature remains neutral with regard to jurisdictional claims in published maps and institutional affiliations. 